\title{
WOMEN BORN PRETERM OR SMALL-FOR-GESTATIONAL AGE (SGA) ARE AT RISK OF SUBSEQUENT GESTATIONAL DIABETES MELLITUS (GDM) AND PRE-ECLAMPSIA
}

\author{
R.A. Rogvi ${ }^{1}$, G. Greisen ${ }^{1}$, P. Damm ${ }^{2}$ \\ ${ }^{1}$ Department of Neonatology, ${ }^{2}$ Center for Pregnant Women with Diabetes, Rigshospitalet, Copenhagen \\ University Hospital, Copenhagen, Denmark
}

Background and aims: Low birthweight is associated with development of GDM, pre-eclampsia as well as type 2 diabetes (T2D) later in life. For T2D the increased risk has been shown to be associated with preterm birth and poor fetal growth, but the mechanisms for GDM and pre-eclampsia remain uncertain.

Methods: We identified all Danish women who gave birth to at least one child in the years 1985-2007. Two cohorts of all Danish women born 1974-1977 $(\mathrm{n}=84.219)$ and 1978-1981 $(\mathrm{n}=32.376)$ were created, due to different methods of registering birth weight and gestational age in the two periods. Data was linked with information on gestational diabetes, pre-eclampsia and maternal educational level.

Results: Mothers born SGA (BW $<-2$ SD of expected) had an increased risk of developing GDM (RR $=$ 1.20 [0.94-1.52] for the first cohort, $\mathrm{RR}=1.84$ [1.21-2.81] for the second cohort), and pre-eclampsia $(\mathrm{RR}=$ $1.60[1.35-1.87]$ and $\mathrm{RR}=1.72[1.26-2.33])$.

In a multivariate logistic regression model the risk of developing gestational diabetes was associated with being born SGA ( $p<0.19$ for 1974-1977, $p=0.044$ for 1978-1981) and low gestational age $(p=0.036$ and $p$ $=0.31)$. The risk of developing pre-eclampsia was associated with being born SGA $(\mathrm{p}<0.0001$ and $\mathrm{p}=$ $0.0001)$ and low gestational age $(\mathrm{p}<0.041$ and $\mathrm{p}=0.10)$.

Conclusion: In this cohort of young Danish mothers, prematurity as well as SGA was associated with increased risks of gestational diabetes and pre-eclampsia. 